\title{
Contents, Vol. 2, 1945
}

\section{Index}

Berblinger, IV., Der intestinale tuberkulöse Primärcomplex beim Erwachßenen 129

Boehm, F., Überempfindlichkeitsreaktionen im Ablauf der menschlichen

Lnngentuberkulose 109

Boss, J., Über sogenannte Riesenzellenpneumonie beim Erwachsenen, zugleich ein Beitrag zur Kenntnis der Periarteriitis nodosa tnberkulößer

Áetiologie 89

Egger, K. und H. Good, Zur Technik der kû́nstUchen Pleuraverödung als Vorbereitung för die Kavernensaugdrainage $\quad 24$

Escher, F., Die Tonsillektomie bei Tuberkulöse $\quad 248$

Gies, J. vide Jeanneret, R.

Good, H. vide Egger, K.

J accord, G., Über die Häufigkeit der Nierentuberkulose

Jeanneret, R. et J. Gies, Frequence et comportement de la tuberculose chez les étudiants en médecine des universités suisses Jéquíer-Doge, Ed., Thoracoplastie et fonction pulmonaire Nícod, J. L, Tuberculose bronchique et hémoptysie 76

Pfisterer, G., Das Wassermann (Wa-R) positive Lnngeninfiltrat .... 139

Rotach, F., Eine Tuberkulose-Endemie in einer Schwachsinnigen-Anstalt 212

Rotach, F., Die Beurteilung der tuberkulösen Lungenherde im Tomogramm

Sommer, E., Endemisches Auftxeten yon eosinophilen Pneumonien ... 34

Sprenger-Cathomas, M., Verlauf und Pathogenese der Hal $\beta$ drǘsentuberkulose bei KJndern 189

Stephaní, J., La notation des observations radioscopiques faites au dispenßaire chez Ies enfants 